\title{
A Log-Ratio Information Measure for Stochastic Sensor Management
}

\author{
Daniel Lyons, Benjamin Noack, and Uwe D. Hanebeck \\ Intelligent Sensor-Actuator-Systems Laboratory (ISAS) \\ Institute for Anthropomatics \\ Karlsruhe Institute of Technology (KIT), Germany \\ lyons@kit.edu,noack@kit.edu, uwe.hanebeck@ieee.org
}

\begin{abstract}
In distributed sensor networks, computational and energy resources are in general limited. Therefore, an intelligent selection of sensors for measurements is of great importance to ensure both high estimation quality and an extended lifetime of the network. Methods from the theory of model predictive control together with information theoretic measures have been employed to pick sensors yielding measurements with high information value. We present a novel information measure that originates from a scalar product on a class of continuous probability densities and apply it to the field of sensor management. Aside from its mathematical justifications for quantifying the information content of probability densities, the most remarkable property of the measure, an analogon of the triangle inequality under Bayesian information fusion, is deduced. This allows for deriving computationally cheap upper bounds for the model predictive sensor selection algorithm and for comparing the performance of planning over different lengths of time horizons.
\end{abstract}

\section{INTRODUCTION}

One of the tasks of a sensor network is to collect data about a distributed physical phenomenon and to process the gathered information in a data sink. Due to energy constraints, not every sensor should be measuring at every time instance. Furthermore, measurements lead to a high communication load, which is very costly in terms of power consumption. Therefore, it is of paramount importance to select only those sensors for sensing that yield the highest information gain. The sensor manager has to determine these sensors, considering that the obtained information usually is corrupted by noise. This can be achieved by employing a model-based approach of predicting the impact of future measurements to the current state estimate and assessing the expected information benefit of certain sensor configurations [1], [2]. The configuration with the highest information gain is selected for sensing, the measurements are fused in the data sink, and at the next time instance, the sensor manager repeats the selection based on the updated state estimate.

There are two main challenges, sensor management has to cope with:

1) The sensor manager has to assess the information gain of a sensor configuration by means of an appropriate information measure. The choice of this measure has a strong impact on the quality of the overall state estimate.

2) The forward-looking sensor manager considers all possible sensor configurations in an exhaustive tree search and evaluates their information gain. The complexity of the model predictive approach makes it difficult to compute the optimal solution under real-time constraints.

There are several methods to meet the first one of these challenges. For linear systems with Gaussian noise, covariancebased objective functions are well suited to determine optimal sensor configurations [3]. On the contrary, in nonlinear cases, when dealing with arbitrary stochastic quantities, information theoretic objective functions depending on the corresponding probability densities are usually considered [4]. The most common information measures are the differential entropy [5] and variations like the Kullback-Leibler divergence [6], the mutual information [7], or the more general concept of $f$-divergence [8]. The differential entropy, which is a generalization of the discrete Shannon entropy [9], is in general not positive for continuous densities and, hence, not consistent with the discrete entropy in the context of information theory. A further development of the differential entropy, the continuous Rényi entropy [10], becomes non-unique through the choice of the order $\alpha$. The Fisher information is a geometric approach to information theory, being a Riemannian metric on parameterized probability densities, viewed as a finite-dimensional manifold. The Fisher information is not applicable to general probability densities, because the assumption of a finite parameterization has to be regarded as a restriction.

To deal with the computational complexity of the exhaustive tree search, approximations by suboptimal pruning strategies have been proposed [11]. Greedy sensor scheduling techniques, only concerned with maximizing the information gain of the next measurement, are another way to keep the complexity of the sensor management feasible [12], [13].

In this paper, we present a novel information measure, the log-ratio information measure, and show its relevance to sensor management. In contrast to the Fisher information, the proposed information measure, although featuring a geometric interpretation, does not depend on a finite-dimensional representation of probability densities. More precisely, this novel measure stems from a norm on a vector space of general continuous probability density functions [14].

The log-ratio information measure meets both of the above challenges in sensor management. It rests firmly on a rigorous mathematical framework and for that reason inconsistencies, 
like negative values of the measure, are circumvented. The geometrical interpretation enables us to derive easily computable bounds on the results of the optimal solution to the sensor selection problem.

This paper is structured as follows. Section II will explain the mathematical formulation of the problem setup of sensor scheduling and recursive state estimation in the Bayesian framework. The conceptual ideas behind the log-ratio information measure and the contributions are explained in Section III. The measure is defined in Section IV and its most important properties will be derived. In Section V, we apply the novel measure to sensor scheduling by formulating an information theoretic objective function based on this measure. Furthermore, we deduce the above mentioned upper bounds from the properties illustrated earlier in Section IV. We conclude our paper with the application of the measure to an example from the field of target tracking in Section VI.

\section{Problem SetuP}

In this paper, we focus on the application of sensor networks to monitor spatially distributed physical phenomena in a cooperative manner.

\section{A. Sensor Selection}

On the one hand, using all available sensor nodes at each time step and fusing their estimates yields the most insight into the system state. On the contrary, the resulting computational and communication load will reduce the overall lifetime of the network significantly. In order to find a tradeoff between maximizing estimation quality and minimizing consumption of resources, it is necessary to use only those sensors that provide the most information. The sensor manager therefore aims at maximizing the expected information gain $\mathrm{E}\left\{R\left(\underline{\boldsymbol{x}}_{k}, \underline{s}_{k}\right)\right\}$ about the internal state $\underline{\boldsymbol{x}}_{k}$ of the observed phenomenon, where $R$ is an information theoretic reward function and $\underline{s}_{k}=\left\{s_{k}^{1}, \ldots, s_{k}^{L}\right\}$ is a configuration of sensors that can be applied at the next timestep. The reward function $R$ judges on the information gain of a particular sequence $\underline{s}_{k}$ depending on the current state estimate $\underline{\boldsymbol{x}}_{k}$. The cumulative reward of a sequence of sensor configurations over a certain time horizon $N$ is given by

$$
V_{k, N}\left(\underline{\boldsymbol{x}}_{k: k+N}, \underline{s}_{k: k+N}\right)=\max _{\underline{s}_{k: k+N}} \mathrm{E}\left\{\sum_{i=k}^{k+N} R\left(\underline{\boldsymbol{x}}_{i}, \underline{s}_{i}\right)\right\},
$$

where the notation $\underline{s}_{k: k+N}=\left(\underline{s}_{k}, \underline{s}_{k+1}, \ldots, \underline{s}_{k+N}\right)$ denotes a sequence of sensor configurations and similarly for $\underline{\boldsymbol{x}}_{k: k+N}$. Thus, by finding a sequence $\underline{s}_{k: k+N}$ that fulfills (1), the sensor manager aims at maximizing the expected cumulative information gain over a certain time horizon. Bellman's principle of dynamic programming [15] can be applied to recursively determine a solution for (1), which is then given as

$$
\begin{aligned}
& \underline{s}_{k: k+N}^{*} \\
& \quad=\arg \max _{\underline{s}_{k}}\left[\mathrm{E}\left\{R\left(\underline{\boldsymbol{x}}_{k}, \underline{s}_{k}\right)+V_{k+1, N}\left(\underline{\boldsymbol{x}}_{k+1: N}, \underline{s}_{k+1: N}\right)\right\}\right] .
\end{aligned}
$$

This solution of the maximization is the sequence of sensor configurations that yield the maximum expected information gain. At each time step, the sensor manager calculates an optimal sequence, selects the first configuration of the sequence for measuring, updates the current estimate of the internal system state, and repeats the planning phase.

\section{B. System and Observation Models}

We assume that the spatial and temporal evolution of the physical quantities is given by a discrete-time nonlinear dynamic system model

$$
\underline{\boldsymbol{x}}_{k+1}=\underline{a}_{k}\left(\underline{\boldsymbol{x}}_{k}, \underline{\boldsymbol{w}}_{k}\right),
$$

where vectorial quantities are indicated by underlining. The system function $\underline{a}_{k}$ maps the state $\underline{\boldsymbol{x}}_{k}$, characterizing the phenomenon at time step $k$, to the system state $\underline{\boldsymbol{x}}_{k+1}$ at the next time step. Perturbations and model uncertainties are subsumed by the system noise $\underline{\boldsymbol{w}}_{k}$. Boldface letters are used to symbolize that these quantities are uncertain in a stochastic sense. The time update can also be written by means of the corresponding probability densities

$$
f_{k+1}^{\mathrm{p}}\left(\underline{x}_{k+1}\right)=\int_{\Omega} f_{k}^{\mathrm{T}}\left(\underline{x}_{k+1} \mid \underline{x}_{k}\right) f_{k}\left(\underline{x}_{k}\right) d \underline{x}_{k},
$$

where $f_{k}^{\mathrm{T}}$ denotes the probability density of the state transition [16]. By this model-based approach, we are in a position to predict the future evolution of the system state.

The predicted density can then be fused at the data sink of the network with observations of the sensor nodes as they become available. We describe the sensing modalities of each sensor $s_{k} \in\{1, \ldots, M\}$ by the measurement model

$$
\underline{\hat{z}}_{s_{k}}=\underline{h}_{s_{k}}\left(\underline{\boldsymbol{x}}_{k}, \underline{\boldsymbol{v}}_{s_{k}}\right) \text {. }
$$

Each observation $\underline{z}_{s_{k}}$ is corrupted by a measurement noise $\underline{\boldsymbol{v}}_{s_{k}}$, which is independent for different sensors. In terms of probability densities, the fusion of the prior or predicted density $f_{k}^{\mathrm{p}}$ with an observation $\underline{\hat{z}}_{s_{k}}$ is performed by applying Bayes' rule

$$
f_{s_{k}}^{\mathrm{e}}\left(\underline{x}_{k}\right)=\frac{f_{s_{k}}^{\mathrm{L}}\left(\underline{\hat{z}}_{s_{k}} \mid \underline{x}_{k}\right) f_{k}^{\mathrm{p}}\left(\underline{x}_{k}\right)}{\int_{\Omega} f_{s_{k}}^{\mathrm{L}}\left(\underline{\hat{z}}_{s_{k}} \mid \underline{x}_{k}\right) f_{k}^{\mathrm{p}}\left(\underline{x}_{k}\right) d \underline{x}_{k}},
$$

where $f_{s_{k}}^{\mathrm{L}}\left(\underline{\hat{z}}_{s_{k}} \mid \underline{x}_{k}\right)$ is the likelihood function.

\section{KEY IDEA}

To select a sensor for sensing, judging on the information value of future measurements is of paramount importance. For this purpose, we consider continuous probability densities as elements of a vector space, which has been defined in [14]. This space comes equipped with an inner product inducing a vector norm. Subsequently, we will utilize this norm as a scalar measure of information on a certain set of probability densities.

The train of thought legitimating this approach is as follows: In a general vector space, a norm quantifies the length of a vector, or alternatively, how far away from the zero element 

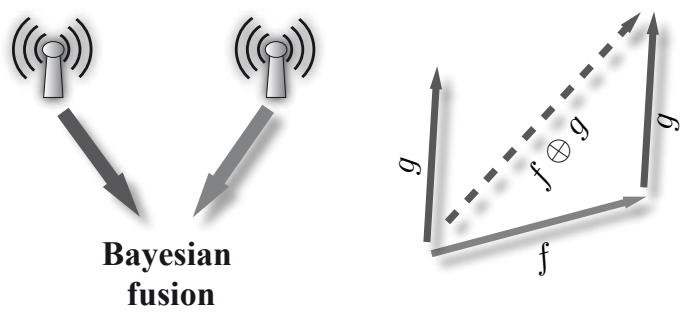

Fig. 1. The information fused from two sensors by applying Bayes' rule (left side) can be interpreted as addition in a vector space (right side).

the vector is. In terms of probability densities, the zero element becomes the uniform density. It characterizes a random quantity with the least information value, since every outcome of the experiment has the same probability of occurance. Accordingly, by taking the norm of a probability density, we rate on how much the density differs from the density of the uniform distribution. Resting upon this mathematical background, certain properties of the proposed information measure, e.g., positiveness, are guaranteed.

Another crucial property, which can be derived from the underlying vector space framework, is an analogon of the triangle inequality in the Euclidean space. In the space of probability densities, the Bayesian data fusion (3) is understood as adding two vectors, depicted in Fig. 1. The triangle inequality for the log-ratio information measure allows us to derive upper bounds on the recursive solution of the model predictive sensor selection algorithm and also allows for relating model predictive planning over different time horizons. For additive Gaussian measurement noise, there exists a closedform solution of these upper bounds, which can be calculated in real time.

\section{INFORMATION MEASURE}

In this section, we are going to give the definition of the log-ratio information measure $\mathbf{N}$ and show its most important properties, which will then be applied to the task of selecting sensors for measurements in Section V.

Since the log-ratio information measure originates from a norm on a space of probability densities, it quantifies the length of a vector in this space. We can interpret it geometrically as a measure of information on probability densities. It is guaranteed that $\mathbf{N}$ is always positive and a triangle inequality holds for the addition defined in this vector space, because $\mathbf{N}$ stems from a true norm.

Definition 1. Let $f: \Omega \rightarrow \mathbb{R}$ be a probability density function, i.e., a non-negative function with unit integral and let $\log (f) \in L^{2}(\Omega)$, where $\Omega$ denotes a probability space with finite measure $\mu(\Omega)$. We define the information measure of $f$ as

$$
\boldsymbol{N}(f):=\sqrt{\int_{\Omega} \int_{\Omega}\left[\log \left(\frac{f(\underline{x})}{f(\underline{y})}\right)\right]^{2} d \underline{x} d \underline{y} .}
$$

Remark 1. Of course, there is some ambiguity involved with the choice of the bounded space $\Omega$, but if it is chosen large enough, all information about the participating densities will be captured. In our definition, we require the logarithm of the density to be square-integrable on $\Omega$. This is to ensure that all integrals in the definition exist. One could drop this condition and just add the constraint, "if all integrals exist", just like in the definition of the differential entropy [5].

Example 1. In order to get an idea of how the log-ratio information measure behaves, let

$$
f(\underline{x})=\frac{1}{2 \pi|C|^{\frac{1}{2}}} \exp \left(-\frac{1}{2} \underline{x}^{\mathrm{T}} C^{-1} \underline{x}\right)
$$

be a two-dimensional axis-aligned multivariate Gaussian density with covariance matrix

$$
C=\left(\begin{array}{cc}
c_{11} & 0 \\
0 & c_{22}
\end{array}\right)
$$

and mean 0 . The square of the log-ratio information measure of $f$ is

$$
\begin{aligned}
\mathbf{N}(f)^{2} & =\int_{\Omega} \int_{\Omega}\left(-\frac{1}{2} \underline{x}^{\mathrm{T}} C^{-1} \underline{x}+\frac{1}{2} \underline{y}^{\mathrm{T}} C^{-1} \underline{y}\right)^{2} d \underline{x} d \underline{y} \\
& =\frac{\mu(\Omega)}{4} \int_{\Omega}\left(\underline{x}^{\mathrm{T}} C^{-1} \underline{x}\right)^{2} d \underline{x} \\
& -\frac{1}{2} \int_{\Omega} \int_{\Omega}\left(\underline{x}^{\mathrm{T}} C^{-1} \underline{x}\right)\left(\underline{y}^{\mathrm{T}} C^{-1} \underline{y}\right) d \underline{x} d \underline{y}
\end{aligned}
$$

with

$$
\begin{aligned}
\int_{\Omega} & \left(\underline{x}^{\mathrm{T}} C^{-1} \underline{x}\right)^{2} d \underline{x} \\
& =\int_{\Omega} \frac{1}{c_{11}^{2}} x_{1}^{4}+\frac{1}{c_{22}^{2}} x_{2}^{4}+\frac{2}{c_{11} c_{22}} x_{1}^{2} x_{2}^{2} d x_{1} d x_{2}
\end{aligned}
$$

and a similar second summand. As the integrals remain constant when we choose $\Omega$ to be symmetrical around zero, maximizing the information gain would correspond to maximizing

$$
\frac{1}{c_{11}^{2}}+\frac{1}{c_{22}^{2}}=\operatorname{trace}\left(C^{-1} \cdot C^{-1}\right)
$$

together with

$$
\frac{1}{c_{11} c_{22}}=\operatorname{det} C^{-1}=\frac{1}{\operatorname{det} C} .
$$

We will now prove the properties for $\mathbf{N}$ mentioned in Section III. The fact that $\mathbf{N}$ is a norm on a certain space of probability density functions follows from the construction via an inner product in [14], [17]. Our proof of the properties we consider here is a standalone version of that argumentation.

Theorem 1. The information measure $\boldsymbol{N}$ from Definition 1 satisfies the following properties for all $f$ and $g$ as in the definition

- $N(f) \geq 0$,

- $N(f)=0$ if and only if $f$ is the uniform distribution on $\Omega$, and

- $\boldsymbol{N}(f \cdot g) \leq \boldsymbol{N}(f)+\boldsymbol{N}(g)$, where $f \cdot g$ is defined via pointwise multiplication of $f$ and $g$. 
For a proof, see Appendix I.

Remark 2. The scaling factor in Bayesian data fusion has no influence on the information measure of the fusion of two probability densities, because we only consider quotients of probability densities in the definition of $\mathbf{N}$. This is the reason, why in Theorem 1 we merely considered $f \cdot g$ and not

$$
\frac{f(\underline{x}) \cdot g(\underline{x})}{\int f(\underline{x}) \cdot g(\underline{x}) d \underline{x}},
$$

as would be the result of the Bayesian data fusion rule (3).

Example 2. The Shannon-entropy of a discrete random variable $X$ with range $\mathcal{H}$ is defined as

$$
H(X):=-\sum_{x \in \mathcal{H}} p(x) \log p(x) .
$$

For the discrete entropy, the uniform distribution on $\mathcal{H}$ forms an upper bound for the entropy of any other random variable $X$ with same range. It holds

$$
H(X) \leq \log |\mathcal{H}|,
$$

where $|\mathcal{H}|$ denotes the number of elements in $\mathcal{H}$ and equality holds if and only if $X$ is the uniform distribution on $\mathcal{H}$ [5]. When generalizing the discrete entropy to continuous probability densities, this bound is no longer true for the differential entropy.

In our case, the information measure is bounded from below by 0 , which is the measure of the uniform distribution on $\Omega$.

Although $\mathbf{N}$ is defined through a double integral, there is a reformulation of the measure in terms of simple integrals. In fact, the reformulation shows where precisely the squareintegrability of the logarithm of the density comes into play.

Theorem 2. Let $f$ be as in Definition 1, then the following equation holds for $\mathrm{N}(f)$

$$
\begin{aligned}
& \boldsymbol{N}(f)^{2}= \\
& \quad 2 \mu(\Omega) \int_{\Omega}[\log f(\underline{x})]^{2} d \underline{x}-2\left[\int_{\Omega} \log f(\underline{x}) d \underline{x}\right]^{2},
\end{aligned}
$$

where $\mu$ is the measure on the probability space $\Omega$.

Proof: The proof follows from expanding

$$
\left[\log \left(\frac{f(\underline{x})}{f(\underline{y})}\right)\right]^{2}
$$

and using the fact that $\Omega$ has finite mass $\mu(\Omega)<\infty$.

\section{SENSOR SCHEduling}

In this section, we apply the log-ratio information measure to the sensor selection problem. For simplicity reasons, we only consider selecting one sensor $s_{k}$ at each time step for measuring, although our approach can be generalized to selecting a subset of sensors for measurement at each time step $k$. This can be achieved by filtering the prior density of the current state estimate with the likelihoods of multiple sensors $\left(s_{k 1}, \ldots, s_{k n}\right)$ following Bayes' rule (3) and taking the expectation over the respective measurements. The following results remain unchanged.

\section{A. Information Gain}

As mentioned in the introduction, the task of the sensor manager is to select sensors for sensing whose measurements have high expected information. Let $f_{k}$ be the density representing the current state estimate. We then denote the posterior density by $f_{s_{k}}^{\mathrm{e}}$, which is obtained by filtering the predicted density $f_{k}^{\mathrm{p}}$ with the likelihood $f_{s_{k}}^{\mathrm{L}}$ of sensor $s_{k}$

$$
f_{s_{k}}^{\mathrm{e}}\left(\underline{x}_{k} \mid \underline{\hat{z}}_{s_{k}}\right):=\frac{1}{c_{k}} f_{k}^{\mathrm{p}}\left(\underline{x}_{k}\right) \cdot f_{s_{k}}^{\mathrm{L}}\left(\underline{\hat{z}}_{s_{k}} \mid \underline{x}_{k}\right)
$$

as described in Section II. For the sake of simplicity, we abbreviate $\underline{z}_{s_{k}}$ by $\underline{z}_{k}$ whenever we consider the expectation over all possible measurements. We describe the expected information gain after filtering the density of the current state estimate with measurements $\underline{z}_{k}$ from sensor $s_{k}$ as

$$
\mathrm{E}_{\underline{z}_{k}}\left\{\mathbf{N}\left(f_{s_{k}}^{\mathrm{e}}\left(\cdot \mid \underline{z}_{k}\right)\right)\right\}=\int \mathbf{N}\left(f_{s_{k}}^{\mathrm{e}}\left(\cdot \mid \underline{z}_{k}\right)\right) f\left(\underline{z}_{k}\right) d \underline{z}_{k} .
$$

Maximizing (5) leads to maximizing the expected information gain in the next time step. Because we do not only want to maximize the gain of information in the next time step, but also anticipate future measurements and maximize the expected information gain over an horizon of length $N$, we apply methods from model predictive control [18].

The recursive equation derived from Bellman's principle of optimality [15], [18] for the expected information gain over $N$ time steps is described by

$$
\begin{aligned}
& V_{k}\left(f_{k}\right):= \\
& \max _{s_{k}}\left[\mathrm{E}_{\underline{z}_{k}}\left\{\mathbf{N}\left(f_{s_{k}}^{\mathrm{e}}\left(\cdot \mid \underline{z}_{k}\right)\right)+V_{k+1}\left(f_{s_{k}}^{\mathrm{e}}\left(\cdot \mid \underline{z}_{k}\right)\right)\right\}\right]
\end{aligned}
$$

with terminal gain

$$
V_{N}\left(f_{k}\right):=\max _{s_{N}}\left[\mathrm{E}_{\underline{z}_{N}}\left\{\mathbf{N}\left(f_{s_{N}}^{\mathrm{e}}\left(\cdot \mid \underline{z}_{N}\right)\right)\right\}\right]
$$

for the last time step. It is important to note that from time step $k+1$ on, we do not predict the state estimate using the Chapman-Kolmogorov equation (2). While this can be done, our emphasis is on the information gain of future measurements.

\section{B. Upper Bounds on the Log-Ratio Measure}

Finding a sensor configuration that maximizes the objective function (6) is in general computationally infeasible when dealing with continuous probability densities and because of the recursive nature of the equation. For real-time applications, it is therefore of high importance to obtain easily computable bounds on the solution of (6) and to keep the recursion depth as moderate as possible with regard to estimation quality. In this section and the next section, we present upper bounds on the value of $V_{k}$ as well as deduce upper bounds for the loss of performance when regarding shorter time horizons in the sensor management algorithm (6). To make the idea behind the calculations clear, we show all results for planning over horizons of length 1 and 2. By induction over the number of planning steps the time horizon can be extended arbitrarily. 
The myopic sensor manager aims at maximizing the expectation of

$$
\mathbf{N}\left(f_{s_{k}}^{\mathrm{e}}\left(\cdot \mid \underline{z}_{k}\right)\right)
$$

over all future measurements. Applying the results of Theorem 1 , we obtain the estimate

$$
\mathbf{N}\left(f_{s_{k}}^{\mathrm{e}}\left(\cdot \mid \underline{z}_{k}\right)\right) \leq \mathbf{N}\left(f_{k}^{\mathrm{p}}\right)+\mathbf{N}\left(f_{s_{k}}^{\mathrm{L}}\left(\underline{z}_{k} \mid \cdot\right)\right)
$$

for the log-ratio information measure. The first summand on the right hand side does not depend on the measurements $\underline{z}_{k}$ anymore. Hence, we can derive the upper bound

$$
\begin{aligned}
\max _{s_{k}} & {\left[\mathrm{E}_{\underline{z}_{k}}\left\{\mathbf{N}\left(f_{s_{k}}^{\mathrm{e}}\left(\cdot \mid \underline{z}_{k}\right)\right)\right\}\right] } \\
& \leq \mathbf{N}\left(f_{k}^{\mathrm{p}}\right)+\max _{s_{k}}\left[\mathrm{E}_{\underline{z}_{k}}\left\{\mathbf{N}\left(f_{s_{k}}^{\mathrm{L}}\left(\underline{z}_{k} \mid \cdot\right)\right)\right\}\right]
\end{aligned}
$$

for the myopic sensor manager.

Now we will provide a similar result for the solution $V_{k, 2}$ of (6) for $N=2$,

$$
\begin{aligned}
& V_{k, 2}\left(f_{k}\right)= \\
& \max _{s_{k}}\left[\mathrm{E}_{\underline{z}_{k}}\left\{\mathbf{N}\left(f_{s_{k}}^{\mathrm{e}}\left(\cdot \mid \underline{z}_{k}\right)\right)+V_{k+1,2}\left(f_{s_{k}}^{\mathrm{e}}\left(\cdot \mid \underline{z}_{k}\right)\right)\right\}\right]
\end{aligned}
$$

with terminal information gain

$$
\begin{aligned}
& V_{k+1,2}\left(f_{s_{k}}^{\mathrm{e}}\left(\cdot \mid \underline{z}_{k}\right)\right)= \\
& \max _{s_{N}}\left[\mathrm{E}_{\underline{z}_{k+1}}\left\{\mathbf{N}\left(f_{s_{k}, s_{k+1}}^{\mathrm{e}}\left(\cdot \mid \underline{z}_{k}, \underline{z}_{k+1}\right)\right)\right\}\right] .
\end{aligned}
$$

For $V_{k+1,2}$, the inequality $\mathbf{N}(f \cdot g) \leq \mathbf{N}(f)+\mathbf{N}(g)$ gives

$$
\begin{aligned}
V_{k+1,2} & \left(f_{s_{k}}^{\mathrm{e}}\left(\cdot \mid \underline{z}_{k}\right)\right) \\
& \leq \max _{s_{k+1}}\left[\mathrm{E}_{\underline{z}_{k}}\left\{\mathbf{N}\left(f_{s_{k}}^{\mathrm{e}}\left(\cdot \mid \underline{z}_{k}\right)\right)\right\}\right. \\
& \left.+\mathrm{E}_{\underline{z}_{k+1}}\left\{\mathbf{N}\left(f_{s_{k+1}}^{\mathrm{L}}\left(\underline{z}_{k+1} \mid \cdot\right)\right)\right\}\right]
\end{aligned}
$$

and as the first summand $\mathbf{N}\left(f_{s_{k}}^{\mathrm{e}}\left(\cdot \mid \underline{z}_{k}\right)\right)$ does not depend on $s_{k+1}$ or $\underline{z}_{k+1}$, we have

$$
\begin{aligned}
V_{k+1,2} & \left(f_{s_{k}}^{\mathrm{e}}\left(\cdot \mid \underline{z}_{k}\right)\right) \\
& \leq \mathbf{N}\left(f_{s_{k}}^{\mathrm{e}}\left(\cdot \mid \underline{z}_{k}\right)\right) \\
& +\max _{s_{k+1}}\left[\mathrm{E}_{\underline{z}_{k+1}}\left\{\mathbf{N}\left(f_{s_{k+1}}^{\mathrm{L}}\left(\underline{z}_{k+1} \mid \cdot\right)\right)\right\}\right] .
\end{aligned}
$$

Insertion of the above result into $V_{k, 2}$ yields

$$
\begin{aligned}
V_{k, 2}\left(f_{k}\right) & \leq \max _{s_{k}}\left[\mathrm{E}_{\underline{z}_{k}}\left\{2 \mathbf{N}\left(f_{s_{k}}^{\mathrm{e}}\left(\cdot \mid \underline{z}_{k}\right)\right)\right\}\right] \\
& +\max _{s_{k+1}}\left[\mathrm{E}_{\underline{z}_{k+1}}\left\{\mathbf{N}\left(f_{s_{k+1}}^{\mathrm{L}}\left(\underline{z}_{k+1} \mid \cdot\right)\right)\right\}\right]
\end{aligned}
$$

for the planning performance with length 2 . The inequality in (10) can be made easier to compute by further estimating

$$
\begin{aligned}
\max _{s_{k}} & {\left[\mathrm{E}_{\underline{z}_{k}}\left\{2 \mathbf{N}\left(f_{s_{k}}^{\mathrm{e}}\left(\cdot \mid \underline{z}_{k}\right)\right)\right\}\right] } \\
& \leq 2 \mathbf{N}\left(f_{k}^{\mathrm{p}}\right)+\max _{s_{k}}\left[\mathrm{E}_{\underline{z}_{k}}\left\{2 \mathbf{N}\left(f_{s_{k}}^{\mathrm{L}}\left(\underline{z}_{k} \mid \cdot\right)\right)\right\}\right],
\end{aligned}
$$

with the same argumentation as for the myopic sensor manager by just applying the inequality for the log-ratio information measure again. The overall bound on $V_{k, 2}$ is

$$
\begin{aligned}
V_{k, 2}\left(f_{k}\right) & \leq 2 \mathbf{N}\left(f_{k}^{\mathrm{p}}\right)+\max _{s_{k}}\left[\mathrm{E}_{\underline{z}_{k}}\left\{2 \mathbf{N}\left(f_{s_{k}}^{\mathrm{L}}\left(\underline{z}_{k} \mid \cdot\right)\right)\right\}\right] \\
& +\max _{s_{k+1}}\left[\mathrm{E}_{\underline{z}_{k+1}}\left\{\mathbf{N}\left(f_{s_{k+1}}^{\mathrm{L}}\left(\underline{z}_{k+1} \mid \cdot\right)\right)\right\}\right] .
\end{aligned}
$$

These bounds can be calculated iteratively for arbitrary recursion depths in the planning algorithm. Note that in the case of additive Gaussian measurement noise, the log-ratio information of the likelihood can be calculated in closed form and the upper bound in (10) is inexpensive to compute even in real-time applications.

If the measurement models for each sensor $s_{k}$ are of the form

$$
\underline{\hat{z}}_{s_{k}}=\underline{h}_{s_{k}}\left(\underline{\boldsymbol{x}}_{k}\right)+\underline{\boldsymbol{v}}_{s_{k}},
$$

with mutually independant additive noise terms $\underline{\boldsymbol{v}}_{s_{k}}$, the probability density of obtaining measurement $\underline{\underline{z}}_{s_{k}}$ conditioned on the system state $\underline{\boldsymbol{x}}_{k}$ for sensor $s_{k}$ is given by

$$
f_{s_{k}}^{\mathrm{L}}\left(\underline{\hat{z}}_{s_{k}} \mid \underline{x}_{k}\right)=f^{v_{s_{k}}}\left(\underline{\hat{z}}_{s_{k}}-\underline{h}_{s_{k}}\left(\underline{x}_{k}\right)\right) \text {. }
$$

In case of white Gaussian noise $\underline{\boldsymbol{v}}_{s_{k}}$, the likelihood simply is

$$
f_{s_{k}}^{\mathrm{L}}\left(\underline{\hat{z}}_{s_{k}} \mid \underline{x}_{k}\right)=\mathcal{N}\left(\underline{\hat{z}}_{s_{k}}-\underline{h}_{s_{k}}\left(\underline{\boldsymbol{x}}_{k}\right), C\right)
$$

and $\mathbf{N}\left(f_{s_{k}}^{\mathrm{L}}\left(\underline{\hat{z}}_{s_{k}} \mid \cdot\right)\right)$ can be calculated as a function of the value $\underline{\hat{z}}_{s_{k}}$ and the measurement model $\underline{h}_{s_{k}}\left(\underline{\boldsymbol{x}}_{k}\right)$, as sketched for a two-dimensional Gaussian density in Example 1.

\section{Sensor Manager Decision Rule}

In this section, we propose a decision rule for a sensor manager based on the estimates in (10) for comparing the performance of the myopic selection algorithm and the planning with horizon length 2 . The myopic sensor manager aims at maximizing

$$
\mathrm{E}_{\underline{z}_{k}}\left\{\mathbf{N}\left(f_{s_{k}}^{\mathrm{e}}\left(\cdot \mid \underline{z}_{k}\right)\right)\right\}
$$

over all possible sensors. The upper bound for the sensor manager with time horizon 2 we deducted in (10) is

$$
\begin{aligned}
V_{k, 2}\left(f_{k}\right) & \leq 2 \max _{s_{k}}\left[\mathrm{E}_{\underline{z}_{k}}\left\{\mathbf{N}\left(f_{s_{k}}\left(\cdot \mid \underline{z}_{k}\right)\right)\right\}\right] \\
& +\max _{s_{k+1}}\left[\mathrm{E}_{\underline{z}_{k+1}}\left\{\mathbf{N}\left(f_{s_{k+1}}^{\mathrm{L}}\left(\underline{z}_{k+1} \mid \cdot\right)\right)\right\}\right]
\end{aligned}
$$

and when calculating the difference to the solution of the greedy planning $V_{k, 1}$, we obtain

$$
V_{k, 2}\left(f_{k}\right)-2 V_{k, 1} \leq \max _{s_{k+1}}\left[\mathrm{E}_{\underline{z}_{k+1}}\left\{\mathbf{N}\left(f_{s_{k+1}}^{\mathrm{L}}\left(\underline{z}_{k+1} \mid \cdot\right)\right)\right\}\right]
$$

as an upper bound for the performance loss when applying the myopic sensor manager.

A sensor manager exploiting these bounds would at time step $k$ compute the upper bound in (11) and make a decision based on this bound whether or not to calculate $V_{k, 2}$ or $V_{k, 1}$. It could be possible to exploit prior knowledge about the 
observed phenomenon and the sensor network by defining a threshold $\varepsilon_{0}$ for the decision rule. If the bound on the expected performance loss for planning over a shorter time horizon is above $\varepsilon_{0}$, the sensor manager should employ the longer planning horizon. If the expected performance loss is below $\varepsilon_{0}$, the loss of perfomance is bounded by $\varepsilon_{0}$ and small enough to still ensure a reasonable estimation quality and hence the computationally cheaper myopic planning can be used, as summarized in Fig. 2. This decision rule enables the sensor manager to avoid the time-consuming recursive scheduling calculations as often as possible.

\section{At each time step $k$ :}

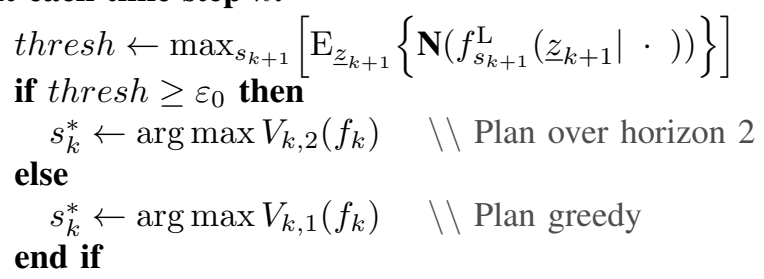

Fig. 2. The sensor manager calculates the bound from (11) and depending on the results, the optimal sensor $s_{k}^{*}$ is determined.

\section{Simulation}

In this section, we evaluate the performance of the log-ratio information measure by applying it to an example from the field of target tracking.

\section{A. Setup}

As an example application, we consider the tracking of a vehicle observed by a small sensor network consisting of distance and bearing sensors over ten time steps [2], [12]. The task of the sensor manager in this scenario is to select one sensor for measuring at each time step. The nonlinear model of the vehicle kinematics is assumed as follows

$$
\left[\begin{array}{l}
\boldsymbol{x}_{k+1} \\
\boldsymbol{y}_{k+1} \\
\boldsymbol{\Phi}_{k+1}
\end{array}\right]=\left[\begin{array}{c}
\boldsymbol{x}_{k} \\
\boldsymbol{y}_{k} \\
\boldsymbol{\Phi}_{k}
\end{array}\right]+\left[\begin{array}{c}
v_{k} \cdot \cos \left(\boldsymbol{\Phi}_{k}\right) \\
v_{k} \cdot \sin \left(\boldsymbol{\Phi}_{k}\right) \\
\alpha_{k}
\end{array}\right]+\left[\begin{array}{c}
\boldsymbol{w}_{k+1}^{x} \\
\boldsymbol{w}_{k+1}^{y} \\
\boldsymbol{w}_{k+1}^{\Phi}
\end{array}\right],
$$

where $\left[\boldsymbol{x}_{k}, \boldsymbol{y}_{k}\right]^{\mathrm{T}}$ denotes the two-dimensional position of the vehicle, $\boldsymbol{\Phi}_{k}$ the vehicle's orientation, $v_{k}$ the speed of the vehicle, $\alpha_{k}$ steering angle applied, and $\underline{\boldsymbol{w}}_{k}$ denotes stochastic additive white Gaussian noise. The initial state estimate is represented by a Gaussian density with mean $\underline{\hat{x}}_{0}=\underline{0}$ and covariance matrix $\mathbf{C}_{0}=\operatorname{diag}\left[\sqrt{5} \mathrm{~m}, \sqrt{5} \mathrm{~m}, \sqrt{\frac{\pi}{2}} \mathrm{rad}\right]$.

The nonlinear observation models for the distance sensors and the bearing sensors are

$$
\hat{z}_{s_{k}}=\sqrt{\left(\boldsymbol{x}_{k}-x_{s_{k}}\right)^{2}+\left(\boldsymbol{y}_{k}-y_{s_{k}}\right)^{2}}+\boldsymbol{v}_{s_{k}}
$$

and

$$
\hat{z}_{s_{k}}=\arctan \left(\frac{\boldsymbol{y}_{k}-y_{s_{k}}}{\boldsymbol{x}_{k}-x_{s_{k}}}\right)+\boldsymbol{v}_{s_{k}}
$$

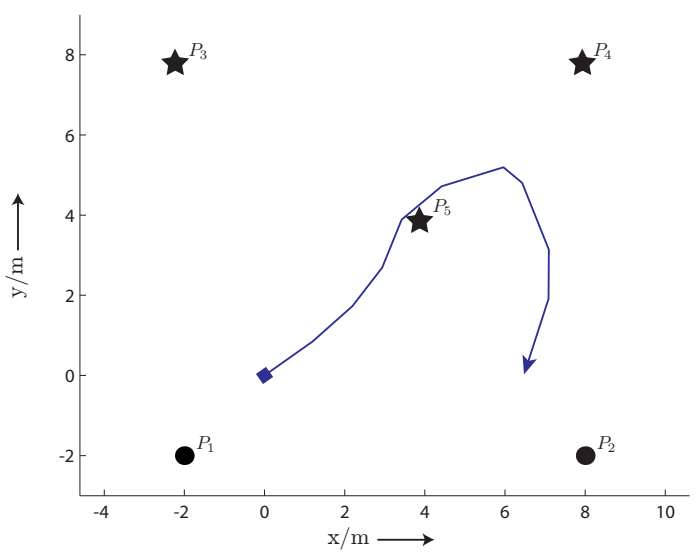

Fig. 3. Sample trajectory of the vehicle. The distance sensors are depicted by circles and the bearing sensors by stars.

respectively, where $\left[x_{s_{k}}, y_{s_{k}}\right]^{\mathrm{T}}$ is the position of sensor $s_{k}$. For the distance sensor, we assume additive white Gaussian noise $\boldsymbol{v}_{s_{k}}$ with standard deviation $\sigma_{s_{k}}=\sqrt{0.2} \mathrm{~m}$ and also for the bearing sensor with standard deviation $\sigma_{s_{k}}=\sqrt{0.01} \mathrm{rad}$. The state estimation is performed by means of the extended Kalman filter.

We compare a myopic sensor manager maximizing the expected information gain in terms of the log-ratio information measure with a sensor manager that selects the sensor maximizing the information gain in terms of the differential entropy [1], [2] and with a sensor manager that randomly selects a sensor for sensing. The log-ratio information measure for the first sensor manager is computed via the Simpson integration rule, using the formula in Theorem 2.

\section{B. Results}

In Fig. 3, a sample trajectory for the vehicle and in Fig. 4, the root-mean-square error (rmse) of the target position for each sensor manager for 30 runs are depicted. The log-ratio manager outperforms both the sensor manager maximizing the entropy and the random manager in terms of the error in the estimation of the target position. In Fig. 5, we compared a myopic sensor manager with a sensor manager using a planning horizon of length two as proposed in Section V. We see that the forward looking sensor manager clearly outperforms the myopic sensor manager and is able to offer a nearly constant estimation quality over several time steps with measurements from only one sensor at each time step.

\section{Choices of the Space $\Omega$}

The choice of the space $\Omega$ may influence the computational complexity of the integration in the definition of the log-ratio information measure. Since we are dealing with logarithms of probability densities, an inadequate choice of $\Omega$ could result in numerical instabilities. In our simulations we have experimented with several different alternatives for $\Omega$.

Instead of a fixed region $\Omega$, we choose $\Omega$ to be of variable size with variable position. For linear systems or for linearized 


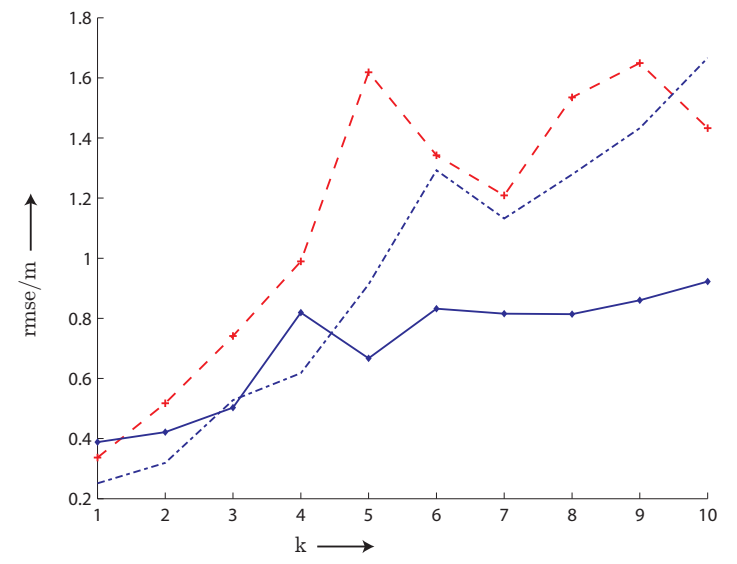

Fig. 4. Average rmse over 30 sample runs. The rmse of the sensor manager employing differential entropy is depicted as a dashed-dotted line, the random manager as a dashed line with cross markers and the sensor manager employing the log-ratio measure as a solid line with diamond markers. All sensor managers planned greedy, i.e., with planning horizon of length one.

systems, we selected $\Omega$ to be a interval of $n$-sigma bounds around the mean of the current predicted state estimate in every dimension. So if the predicted estimate in the above example is characterized by a mean and covariance

$$
\underline{\hat{x}}_{k}^{\mathrm{p}}=\left[\begin{array}{c}
\hat{x}_{k}^{\mathrm{p}} \\
\hat{y}_{k}^{\mathrm{p}} \\
\hat{\Phi}_{k}^{\mathrm{p}}
\end{array}\right], \mathbf{C}_{k}^{\mathrm{p}}=\left[\begin{array}{lll}
c_{11} & c_{12} & c_{13} \\
c_{21} & c_{22} & c_{23} \\
c_{31} & c_{32} & c_{33}
\end{array}\right],
$$

we define

$$
\begin{aligned}
& \Omega_{1}:=\left[\hat{x}_{k}^{\mathrm{p}}-n \cdot c_{11}, \hat{x}_{k}^{\mathrm{p}}+n \cdot c_{11}\right], \\
& \Omega_{2}:=\left[\hat{y}_{k}^{\mathrm{p}}-n \cdot c_{22}, \hat{y}_{k}^{\mathrm{p}}+n \cdot c_{22}\right], \\
& \Omega_{3}:=\left[\hat{\Phi}_{k}^{\mathrm{p}}-n \cdot c_{33}, \hat{\Phi}_{k}^{\mathrm{p}}+n \cdot c_{33}\right],
\end{aligned}
$$

and $\Omega:=\Omega_{1} \times \Omega_{2} \times \Omega_{3}$ with a suitable choice of $n$. In our simulations, $n=3$ gave the best results with the best numerical stability.

\section{CONCLUSIONS AND FUTURE WORK}

We have introduced a novel geometrically motivated information measure and shown its advantages over measures of information derived, e.g., from the differential entropy. The log-ratio information measure is a generalization of the concept of determining lengths of vectors to the infinitedimensional space of probability density functions. Because of its geometrical foundation, it fulfills a variant of the triangle inequality and it is ensured that the log-ratio information measure is always positive. We have applied the log-ratio information measure to the field of sensor management and derived upper bounds on the information gain in a recursive sensor selection algorithm. Furthermore, we have formulated an easily computable decision rule for the sensor manager on how to judge on the loss of estimation quality when planning over shorter time horizons.

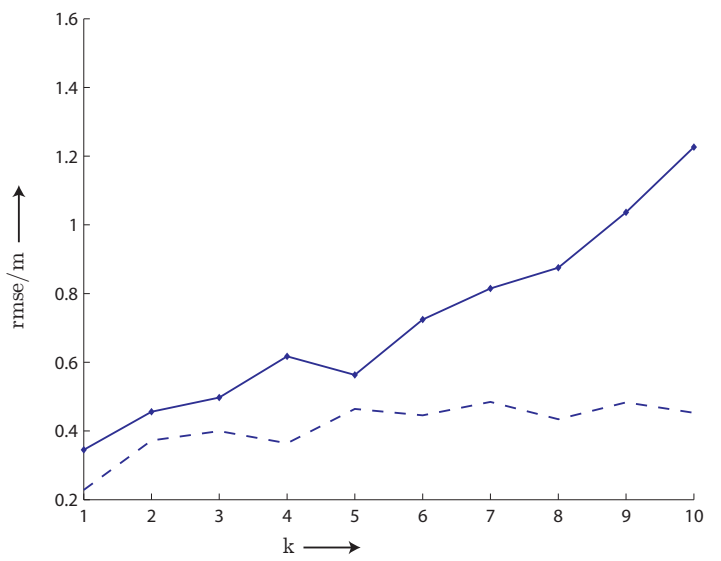

Fig. 5. Average rmse over 50 sample runs. A greedy sensor manager maximizing the expected log-ratio information gain (solid line) is compared with a sensor manager maximizing the information gain in the log-ratio information measure over a planning horizon of length 2 (dashed line).

Future work will be concerned with applying the logratio information measure to decentralized sensor management approaches [19]. The estimate in (9) offers a distinction between the current state estimate and the information value of the measurements. In a decentralized sensor network, each sensor could have a different state estimate and could also lack knowledge about all possible sensor configurations for sensing. This estimate allows to deal with both cases separately, so it is possible to find bounds on the estimation error and also on the planning error made by each sensor.

In real applications with nonlinear system and observation models, the probability densities of uncertain quantities can not be processed efficiently in the Bayesian framework. It is therefore necessary to approximate these densities by more tractable ones. There are several different methods of approximating densities and the particular choice of the approximation is of high relevance to the quality of state estimation. It is therefore important to consider the actual approximation, and the errors made by it, in the sensor selection algorithm. With a metric constructed from the log-ratio information measure, it is possible to assess the quality of the sensor manager with respect to concrete realizations of the employed state estimation procedure.

Finding efficient approximations of the log-ratio information measure will be subject to further research. The numerical integration in the definition of the log-ratio information measure may be be too time-consuming for real-world applications, where decisions have to be made in tenths of seconds by possibly low power embedded systems. Particle methods like the ones proposed in [20], [21], [22] will offer ways to reduce the complexity of the calculations. 


\section{APPENDIX I}

\section{PROOF OF THEOREM 1:}

Since $\left[\log \left(\frac{f(\underline{x})}{f(\underline{y})}\right)\right]^{2} \geq 0$ a.e. for all $f$ as in definition 1 , we have $\mathbf{N}(f) \geq 0$.

Let $\mathbf{N}(f)=0$, then $\left[\log \left(\frac{f(\underline{x})}{f(\underline{y})}\right)\right]^{2}=0$ a.e. on $\Omega$ and it follows that

$$
0=\log \left(\frac{f(\underline{x})}{f(\underline{y})}\right) \text { a.e. }
$$

and hence $\frac{f(\underline{x})}{f(\underline{y})}=1$ a.e. on $\Omega \times \Omega$. Thus, we obtain $f(\underline{x})=f(\underline{y})$ almost everywhere and so $f$ is constant on $\Omega$. Because we demanded the densities to have unit integral, we can conclude that $f$ is the uniform distribution on $\Omega$. Furthermore, the triangle inequality

$$
\begin{aligned}
\mathbf{N}(f \cdot g)^{2}= & \int_{\Omega} \int_{\Omega}\left[\log \left(\frac{f(\underline{x}) g(\underline{x})}{f(\underline{y}) g(\underline{y})}\right)\right]^{2} d \underline{x} d \underline{y} \\
= & \int_{\Omega} \int_{\Omega}\left[\log \left(\frac{f(\underline{x})}{f(\underline{y})}\right)+\log \left(\frac{g(\underline{x})}{g(\underline{y})}\right)\right]^{2} d \underline{x} d \underline{y} \\
= & \mathbf{N}(f)^{2}+\mathbf{N}(g)^{2} \\
& +2 \int_{\Omega} \int_{\Omega} \log \left(\frac{f(\underline{x})}{f(\underline{y})}\right) \cdot \log \left(\frac{g(\underline{x})}{g(\underline{y})}\right) d \underline{x} d \underline{y} \\
& a) \\
\leq & (\mathbf{N}(f)+\mathbf{N}(g))^{2}
\end{aligned}
$$

holds for $\mathbf{N}$, where the inequality a) follows from the fact that

$$
\int_{\Omega} \int_{\Omega} \log \left(\frac{f(\underline{x})}{f(\underline{y})}\right) \cdot \log \left(\frac{g(\underline{x})}{g(\underline{y})}\right) d \underline{x} d \underline{y} \leq \mathbf{N}(f) \cdot \mathbf{N}(g),
$$

which is proven analogously to the Cauchy-Schwarz inequality in general Hilbert spaces [23]. We assume that both $f \neq 1$ and $g \neq 1$, since if for example $f=1$ we would have that $\log \left(\frac{f(x)}{f(y)}\right)=0$ and hence $\mathbf{N}(f)=0$ and the inequality holds. Let $\alpha:=\mathbf{N}(f)$ and $\beta:=\mathbf{N}(g)$ and because of the above assumption we have $\alpha \neq 0$ and $\beta \neq 0$. From

$$
\begin{aligned}
0 & \leq \mathbf{N}\left(\frac{f^{1 / \alpha}}{g^{1 / \beta}}\right)^{2} \\
& =\int_{\Omega} \int_{\Omega}\left[\log \left(\frac{f^{1 / \alpha}(x) g^{1 / \beta}(y)}{f^{1 / \alpha}(y) g^{1 / \beta}(x)}\right)\right]^{2} d \underline{x} d \underline{y} \\
& =\frac{\mathbf{N}(f)^{2}}{\alpha^{2}}-\frac{2}{\alpha \beta} \int_{\Omega} \int_{\Omega} \log \left(\frac{f(\underline{x})}{f(\underline{y})}\right) \cdot \log \left(\frac{g(\underline{x})}{g(\underline{y})}\right) d \underline{x} d \underline{y} \\
& +\frac{1}{\beta^{2}} \mathbf{N}(g)^{2}
\end{aligned}
$$

it follows that

$$
\begin{gathered}
2 \int_{\Omega} \int_{\Omega} \log \left(\frac{f(\underline{x})}{f(\underline{y})}\right) \cdot \log \left(\frac{g(\underline{x})}{g(\underline{y})}\right) d \underline{x} d \underline{y} \\
\quad \leq \frac{\beta}{\alpha} \mathbf{N}(f)^{2}+\frac{\alpha}{\beta} \mathbf{N}(g)^{2}=2 \mathbf{N}(f) \mathbf{N}(g)
\end{gathered}
$$

holds and the proof is complete.

\section{ACKNOWLEDGMENT}

This work was partially supported by the German Research Foundation (DFG) within the Research Training Group GRK 1194 "Self-organizing Sensor-Actuator-Networks".

\section{REFERENCES}

[1] A. O. Hero, C. M. Kreucher, and D. Blatt, Foundations and Applications of Sensor Management, ser. Signals and Communication Technology. Springer, 2008, ch. Information Theoretic Approaches to Sensor Management, pp. 33-59.

[2] M. F. Huber, "Probabilistic Framework for Sensor Management," Ph.D. dissertation, Universität Karlsruhe (TH), 2009.

[3] D. Uciński, Optimal Measurement Methods for Distributed Parameter System Identification, ser. Systems and control series. CRC Press, 2005.

[4] J. L. Williams, "Information Theoretic Sensor Management," Ph.D. dissertation, Massachusetts Institute of Technology, Feb. 2007.

[5] T. M. Cover and J. A. Thomas, Elements of Information Theory, ser Wiley Series in Telecommunications and Signal Processing. WileyInterscience, 2006.

[6] S. Kullback and R. A. Leibler, "On Information and Sufficiency," Annals of Mathematical Statistics, vol. 22, no. 2, pp. 79-86, 1951.

[7] E. Ertin, J. W. Fisher, and L. C. Potter, "Maximum Mutual Information Principle for Dynamic Sensor Query Problems," in Information Processing in Sensor Networks (IPSN), 2003, pp. 405-416.

[8] I. Csiszar, "Information-type Measures of Difference of Probability Distributions and Indirect Observations," Studia. Sci. Math. Hungar. vol. 2, pp. 299-318, 1967.

[9] C. E. Shannon, The Mathematical Theory of Communication. Urbana: Univ. of Ill. Press, 1949

[10] A. O. Hero, B. Ma, O. Michel, and J. Gorman, "Alpha Divergence for Classification, Indexing and Retrieval," University of Michigan, Tech. Rep., 2001.

[11] P. Alriksson and A. Rantzer, "Sub-Optimal Sensor Scheduling with Error Bounds," in Proceedings of the 16th IFAC World Congress, Jul. 2005.

[12] F. Zhao, J. Shin, and J. Reich, "Information-driven Dynamic Sensor Collaboration for Tracking Applications," IEEE Signal Processing Magazine, vol. 19, no. 2, pp. 61-72, 2002.

[13] Y. Oshman, "Optimal Sensor Selection Strategy fo Discrete-Time State Estimators," IEEE Transactions on Aerospace and Electronic Systems, vol. 30, no. 2, pp. 307-314, Apr. 1994.

[14] J. J. Egozcue, J. L. Diaz-Barrero, and V. Pawlosky-Glahn, "Hilbert Space of Probability Density Functions Based on Aitchison Geometry," Acta Mathematica Sinica, English Series, vol. 22, no. 4, pp. 1175-1182, July 2006.

[15] R. E. Bellman, "On the Theory of Dynamic Programming," Proceedings of the National Academy of Sciences, vol. 38, pp. 716-719, 1952.

[16] A. Papoulis and S. U. Pillai, Probability, Random Variables and Stochastic Processes. McGraw-Hill, 2002.

[17] J. J. Egozcue, V. Pawlowsky-Glahn, G. Mateu-Figueras, and C. BarcelóVidal, "Isometric Logratio Transformations for Compositional Data Analysis," Mathematical Geology, vol. 35, no. 3, pp. 279-300, 2003.

[18] D. P. Bertsekas, Dynamic Programming and Optimal Control. Athena Scientific, 2007.

[19] D. S. Bernstein, R. Givan, N. Immerman, and S. Zilberstein, "The Complexity of Decentralized Control of Markov Decision Processes," Math. Oper. Res., vol. 27, no. 4, pp. 819-840, 2002.

[20] S. Singh, N. Kantas, B. Vo, A. Doucet, and R. Evans, "Simulation-based Optimal Sensor Scheduling with Application to Observer Trajectory Planning," Automatica, vol. 43, no. 5, pp. 817-830, 2007.

[21] L. Blackmore and B. Williams, "Optimal, Robust Predictive Control of Nonlinear Systems under Probabilistic Uncertainty using Particles," in American Control Conference, 2007. ACC'07, 2007, pp. 1759-1761.

[22] G. Hoffmann and C. Tomlin, "Mobile Sensor Network Control Using Mutual Information Methods and Particle Filters," IEEE Transactions on Automatic Control, 2009.

[23] K. Yosida, Functional Analysis, ser. Springer Classics in Mathematics. Springer, 2003. 